\title{
Composição da comunidade de Formicidae (Insecta, Hymenoptera) em copas de Attalea phalerata Mart. (Arecaceae), no Pantanal de Poconé, Mato Grosso, Brasil
}

\author{
Leandro Dênis Battirola ${ }^{1,5}$, Marinêz Isaac Marques², Joachim Adis ${ }^{3}$ \& Jacques H. C. Delabie ${ }^{4,6}$
}

${ }^{1}$ Departamento de Zoologia, Universidade Federal do Paraná, Programa de Pós-Graduação em Ciências Biológicas (Entomologia). Caixa Postal 19020,81531-980 Curitiba-PR, Brasil.1dbattirola@uol.com.br

${ }^{2}$ Departamento de Biologia e Zoologia, Instituto de Biociências, Universidade Federal de Mato Grosso, Av. Fernando Corrêa da Costa, s/n, Coxipó, 78060-900 Cuiabá-MT, Brasil.m.marque@terra.com.br

${ }^{3}$ Max-Planck-Institute for Limnology, Tropical Ecology Working Group, Postfach 165, D-24302 Plön, Alemanha. adis@mpil-ploen.mpg.de ${ }^{4}$ Laboratório de Mirmecologia, Convênio UESC/CEPLAC, Centro de Pesquisas do Cacau e Departamento de Ciências Agrárias e

Ambientais, Universidade Estadual de Santa Cruz, Caixa Postal 7, 45600-000 Itabuna-BA, Brasil. delabie@cepec.gov.br

${ }^{5}$ Bolsista CAPES.

${ }^{6} \mathrm{Bolsista} \mathrm{CNPq}$

\begin{abstract}
Composition of Formicidae community (Insecta, Hymenoptera) in the canopy of Attalea phalerata Mart. (Arecaceae), in the Pantanal of Poconé, Mato Grosso, Brazil. Three individuals of the palm Attalea phalerata Mart. (Arecaceae) were sampled using the method of canopy fogging, during the aquatic phase (high water) in the Pantanal of Mato Grosso (February 2001). The objective was to evaluate the diversity, feeding habits and spatial distribution of the Formicidae community in the canopy of this monodominant palm species, typical of this region. Each of three palms was fogged once, then resampled first by shaking fronds while attached, then by washing all fronds (cut then washed). A total of 966 ants belonging to 6 subfamilies, 13 tribes and 29 species was obtained in the $49 \mathrm{~m}^{2}$ sampling area $(19.7 \pm 52.7$ individuals $/ \mathrm{m}^{2}$ ) representing $3.9 \%$ of all arthropods sampled. Myrmicinae was the most representative subfamily, with 6 tribes and 14 species, with Solenopsidini (5 species) distinguishing. Formicinae was the second most abundant subfamily, with 3 tribes and 8 species. Pheidole sp. 2 was dominant in the total catch (284 individuals; $29.4 \%$ of the total captured) followed by Camponotus (Myrmobrachys) crassus (182 individuals; 18.8\%) and Crematogaster (Orthocrema) sp. $1 \mathrm{nr}$. quadriformis (119 individuals; $12.3 \%)$. The values of the diversity indices were significant $\left(\mathrm{H}^{\prime}=2.185 ; \mathrm{D}=0.835\right)$, although they showed low values of equitability ( 0.649 and 0.248 , respectively). This demonstrated the heterogeneity of the Formicidae community associated with the canopy of this palm. An analysis of spatial distribution showed that the greatest abundance and richness of Formicidae occurred in the central region of the crown, next to the trunk.
\end{abstract}

Keywords. Attalea phalerata; canopy; diversity; Formicidae; Pantanal.

Resumo. Composição da comunidade de Formicidae (Insecta, Hymenoptera) em copas de Attalea phalerata Mart. (Arecaceae), no Pantanal de Poconé, Mato Grosso, Brasil. Três indivíduos da palmeira Attalea phalerata Mart (Arecaceae) foram amostrados durante a fase aquática (cheia) no Pantanal de Mato Grosso (fevereiro/2001) utilizando-se a metodologia de nebulização de copas "canopy fogging". Este estudo objetivou avaliar a diversidade, hábitos alimentares e a distribuição espacial da comunidade de Formicidae em copas dessa palmeira que forma adensamentos monodominantes, típicos nessa região. Cada palmeira foi nebulizada uma única vez e realizadas três coletas subseqüentes. Um total de 966 formigas, pertencentes a 6 subfamílias, 13 tribos e 29 espécies foram amostradas em $49 \mathrm{~m}^{2}$ de área amostral $(19,7 \pm 52,7$ indivíduos/ $\mathrm{m}^{2}$ ) representando 3,9\% do total de artrópodes obtidos. Myrmicinae foi a subfamília mais representativa, com 6 tribos e 14 espécies, destacando-se Solenopsidini com 5 espécies. Formicinae foi a segunda subfamília mais abundante com 3 tribos e 8 espécies. Pheidole sp. 2 foi dominante na amostragem geral (284 indivíduos; 29,4\% da captura total) seguida por Camponotus (Myrmobrachys) crassus (182 individuos; 18,8\%) e Crematogaster (Orthocrema) sp. 1 pr. quadriformis (119 individuos; $12,3 \%)$. Os índices de diversidade foram consideráveis $\left(\mathrm{H}^{\prime}=2,185 ; \mathrm{D}=0,835\right)$ apesar de demonstrarem baixos valores de equitabilidade $(0,649$ e 0,248 , respectivamente). Este resultado demonstra a heterogeneidade da comunidade de Formicidae associada à copa dessa palmeira. A análise da distribuição espacial desmonstrou que a maior abundância e riqueza de Formicidae ocorrem na região central da copa, próximo ao caule.

Palavras-Chave. Attalea phalerata; copas; diversidade; Formicidae; Pantanal.

Estudos de artrópodes associados às copas de florestas tropicais têm sido de suma importância para realizar-se uma estimativa do número total de espécies animais sobre o planeta (Erwin 1982). Dentre esses organismos, as formigas merecem destaque por serem consideradas dominantes em número e biomassa nesses ambientes (Adis \& Schubart 1984; Adis et al. 1984; Battirola et al. 2004a; Davidson 1997; Erwin 1983, 1989; Fitkau \& Klinge 1973; Floren \& Linsenmair 1997, 2000;
Harada \& Adis 1997, 1998; Santos et al. 2003; Vasconcelos 1990).

Essa dominância deve-se à forma com que esses organismos têm colonizado o meio terrestre, ocupando os nichos disponíveis, exercendo diversas funções ecológicas e mantendo estreitas associações com outros táxons (Cuezzo 1998; Delabie 2001; Fowler et al. 1991; Hölldobler \& Wilson 1990; Tobin 1995). 


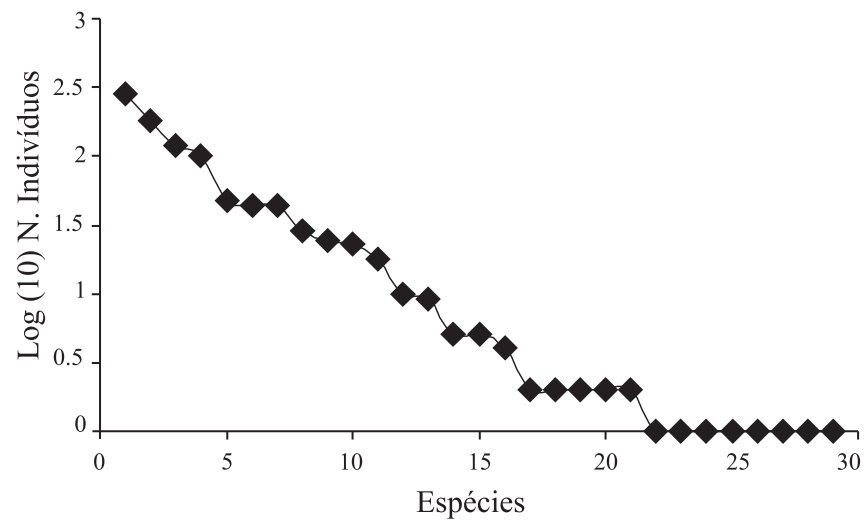

Fig. 1. Distribuição da abundância das espécies para a comunidade de Formicidae em três copas de A. phalerata Mart. (Arecaceae), durante o período de cheia no Pantanal de Poconé, Mato Grosso.

Dentre as funções desempenhadas pelas formigas dentro das comunidades de artrópodes, destaca-se a sua influência sobre a estrutura trófica, atuando como predadores (Floren et al. 2002), consumidores primários e secundários ou como base alimentar para outros consumidores (Cuezzo 1998; Fowler et al. 1991; Tillberg \& Breed 2004). Além disso, apresentam estratégias de forrageamento, hábitos de colonização e padrões diferenciados de organização das colônias (Tobin 1995).

Com relação ao número de espécies, a fauna de formigas da América do Sul é considerada a mais rica do mundo, caracterizada, principalmente, pelo predomínio das subfamílias Myrmicinae, Dolichoderinae e Ponerinae e por um elevado grau de endemismo ao nível genérico, com destaque para os gêneros pertencentes à tribo Attini e outros como Odontomachus Latreille 1804, Crematogaster Lund 1831, Camponotus Mayr 1861, Hypoponera Santschi 1938 e Pheidole Westwood 1841, considerados cosmopolitas, ainda que possam estar representados por espécies particulares em cada região (Cuezzo 1998).

Embora várias espécies de árvores tenham sido investigadas, estudos de comunidades de Formicidae associadas a palmeiras (Vasconcelos 1990) e em ambientes alagáveis (Majer \& Delabie 1994; Santos et al. 2003) são bastante escassos, sendo que os realizados nesses ambientes enfocam comunidades associadas a outros hospedeiros vegetais, podendo-se citar aqueles efetuados na Amazônia por Adis et al. (1984); Adis et al. (1998b); Erwin (1983); Erwin \& Adis (1982); Harada \& Adis (1998), Hurtado-Guerrero et al. (2003) e no Pantanal mato-grossense por Marques et al. (2001).

Desta maneira, considerando a importância das comunidades de formigas arborícolas e o papel desempenhado nesses habitats, essa pesquisa objetivou avaliar a composição, diversidade, hábitos alimentares e distribuição espacial da comunidade de Formicidae, em copas de Attalea phalerata Mart. (Arecaceae) no Pantanal mato-grossense, durante a fase aquática (cheia) dessa região, em áreas onde essa espécie vegetal forma adensamentos monodominantes denominados regionalmente de "acurizais".

\section{MATERIAL E MÉTODOS}

Área de Estudo. O estudo foi realizado no Pantanal de Cuiabá-Bento Gomes-Paraguaizinho, denominado Pantanal de Poconé, na localidade de Pirizal, entre os paralelos $16^{\circ} 15^{\prime} 24^{\prime \prime} \mathrm{e}$ 1754’32"S e meridianos 56³6’24" e 5756’23"W, município de Nossa Senhora do Livramento-Mato Grosso. Essa região é caracterizada por apresentar estações bem definidas, com o período chuvoso de outubro a abril, e a inundação entre dezembro e março (0,6-1,5 m de altura), caracterizando a fase aquática desse ecossistema (Heckmann 1998). Em 2001, período de coleta, a inundação ocorreu de fevereiro a abril e as palmeiras amostradas localizavam-se a uma distância de 30-50 $\mathrm{m}$ do corpo d'água mais próximo.

Metodologia. Três copas de $A$. phalerata Mart. (Arecaceae) foram nebulizadas empregando-se o método de termonebulização de copas ("canopy fogging"), durante o período de cheia (fevereiro/2001), utilizando-se piretróides sintéticos (Lambdacialotrina a 0,5\%).

A seleção das palmeiras amostradas seguiu os critérios propostos por Adis et al. (1998a), e os procedimentos de nebulização e coletas aqueles apresentados por Battirola et al. (2004b). Para a coleta as palmeiras tiveram todo seu diâmetro na base circundado por 16-17 funis de nylon (1m de diâmetro cada), de acordo com a abrangência da copa. Esses funis continham em suas bases frascos de plástico com álcool a $92 \%$, numerados e mapeados, possibilitando a localização do ponto de coleta e a análise de distribuição espacial. Para essa avaliação utilizaram-se apenas os dados referentes a primeira e segunda coletas. As medidas de altura, DAP e diâmetro da copa são apresentados na Tabela I. A palmeira III destacou-se em relação às demais devido a maior quantidade de matéria orgânica associada à copa.

Os artrópodes coletados foram transportados em frascos contendo álcool a 92\% para o Laboratório de Entomologia (21A) do Instituto de Biociências da Universidade Federal de Mato Grosso, onde permanecem devidamente acondicionados. Em laboratório, as formigas foram triadas e separadas dos demais táxons e identificadas por árvore amostrada, número da coleta e funil.

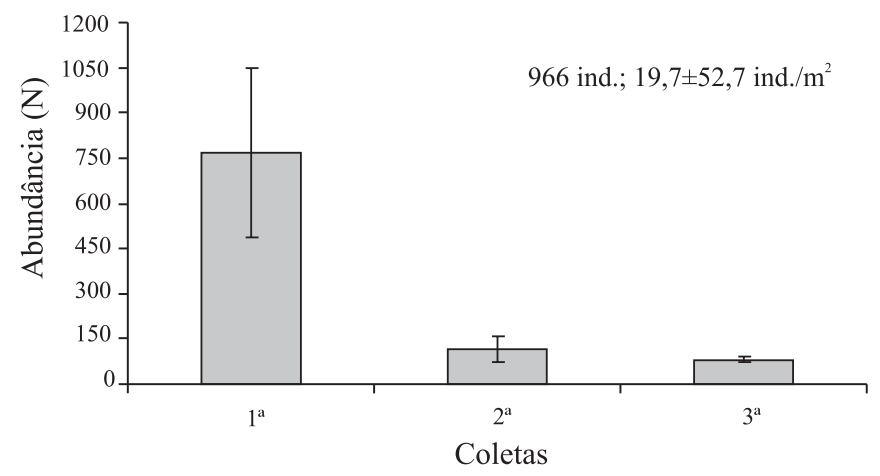

Fig. 2. Abundância de Formicidae, obtida nos três eventos de coleta realizados em três copas de $A$. phalerata Mart. (Arecaceae), durante o período de cheia no Pantanal de Poconé, Mato Grosso. 


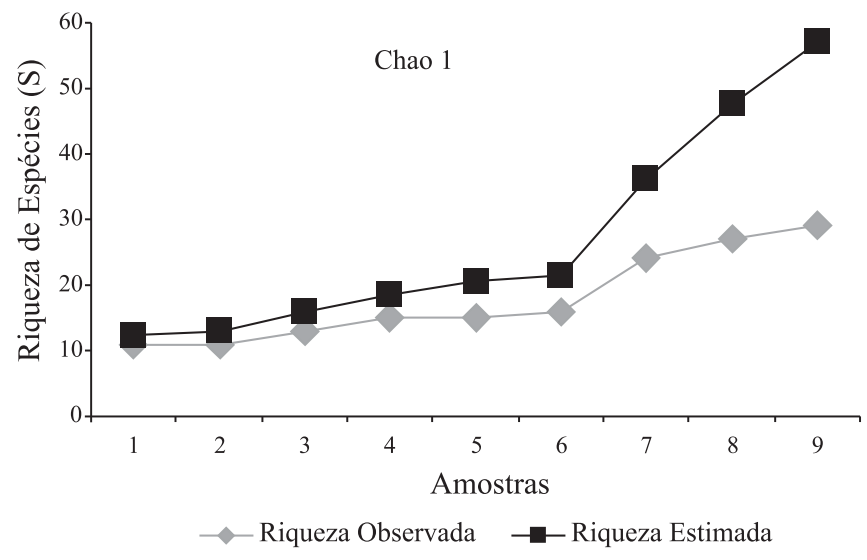

Fig. 3. Comparação entre a riqueza de espécies obtidas e a riqueza estimada (Chao 1), para a comunidade de Formicidae associada a copa de A. phalerata Mart. (Arecaceae), durante o período de cheia no Pantanal de Poconé, Mato Grosso.

Para avaliação da comunidade de Formicidae aplicou-se o índice de similaridade de Sørenson e os índices de diversidade de Shannon-Wiener (H') e Simpson (D), log base “e", de acordo com Magurran (1988). A riqueza de espécies foi avaliada utilizando-se os estimadores Chao 1 e Jack-Knife 1, através do programa Biodiversity Pro. Os hábitos alimentares foram identificados seguindo-se o padrão proposto por Brown (1973) in Fowler et al. (1991).

O material testemunho encontra-se depositado no Laboratório de Entomologia (21A) da Universidade Federal de Mato Grosso, Cuiabá - MT e no Laboratório de Mirmecologia do Centro de Pesquisas do Cacau, CEPLAC, Ilhéus - BA.

\section{RESULTADOS EDISCUSSÃO}

Composição e diversidade da comunidade. Em três copas de A. phalerata foram coletados 966 indivíduos de Formicidae em $49 \mathrm{~m}^{2}$ de área coberta $\left(19,7 \pm 52,7\right.$ indivíduos $\left./ \mathrm{m}^{2}\right)$, distribuídos em 6 subfamílias, 13 tribos e 29 espécies (Tabela II). Verifica-se que muitas espécies amostradas apresentaram baixa abundância, 5 espécies com apenas 2 indivíduos e em 8 apenas 1 indivíduo, representando $44,8 \%$ da riqueza total amostradas (Fig. 1). As formigas corresponderam a 3,9\% do total de artrópodes coletados e a densidade variou entre 3,4 e 41,8 indivíduos $/ \mathrm{m}^{2}$. A maior abundância ocorreu na palmeira III provavelmente devido à maior quantidade de matéria orgânica associada às bainhas foliares (670 indivíduos; 69,3\% da captura total) e a menor abundância na palmeira II (58 indivíduos; 3,4\%). O índice de Sørenson demonstrou que as palmeiras I e II possuem a maior similaridade entre as espécies capturadas $(0,66)$, seguida pelas de número I e III $(0,57)$, e a menor similaridade ocorreu entre as palmeiras II e III $(0,50)$, indicando a heterogeneidade faunística entre as copas (Tabela III).

Do total coletado, 768 indivíduos $(79,5 \%)$, correspondem à primeira coleta, 116 indivíduos (12,0\%) à segunda e $82(8,5 \%)$ à terceira (Fig. 2; Tabela II). Esses valores demonstram que apesar da eficácia da metodologia aplicada, vários indivíduos permanecem presos à copa ou em seus ninhos após a nebulização, justificando a necessidade de sacudir os galhos das palmeiras antes da segunda coleta.

A subfamília mais representativa foi Myrmicinae com 6 tribos (Cephalotini, Attini, Crematogastrini, Solenopsidini, Pheidolini e Dacetini) e 14 espécies, seguida por Formicinae com 3 tribos (Lasiini, Brachymyrmecini e Camponotini) e 8 espécies (Tabelas II e IV). Dentre as tribos de Myrmicinae, Solenopsidini se destaca com 5 espécies do gênero Solenopsis Westwood, 1841. Dentre estas, encontra-se S. saevissima (Fr. Smith, 1855) (23 indivíduos; 2,4\%) que, segundo Adis et al. (2001), durante as inundações no Pantanal mato-grossense apresenta como estratégia de sobrevivência, migrações verticais para ramos de Setaria geniculata (Lam.) Beauv. (Gramineae), planta típica nos campos inundáveis. Este é o primeiro registro da ocorrência dessa espécie terrícola nas copas de $A$. phalerata, o que pode caracterizar sua migração vertical durante a fase aquática (cheia).

Pheidole sp. 2 foi dominante na amostragem geral com 284 indivíduos $(29,4 \%)$, apesar de ter apresentado uma baixa frequência (33,3\%). Apesar disso, não se deve inferir que esta seja a espécie dominante nesse habitat, pois a amostra corresponde àqueles indivíduos que caíram nos funis coletores, sendo que muitos podem ter permanecido presos nas bainhas foliares ou na matéria orgânica existente na copa, e portanto, ser insuficientemente amostradas. Além dessa espécie, Camponotus (Myrmobrachys) crassus Mayr, 1862 (182 indivíduos; 18,8\%), Brachymyrmex sp. (119 indivíduos; $12,3 \%$ ) e Crematogaster (Orthocrema) sp.1 pr. quadriformis (101 indivíduos; 10,5\%) também foram representativas (Tabela II).

Quanto às freqüências, observa-se que grande parte das espécies foi obtida em uma única árvore, demonstrando a heterogeneidade na composição dessa comunidade, condição

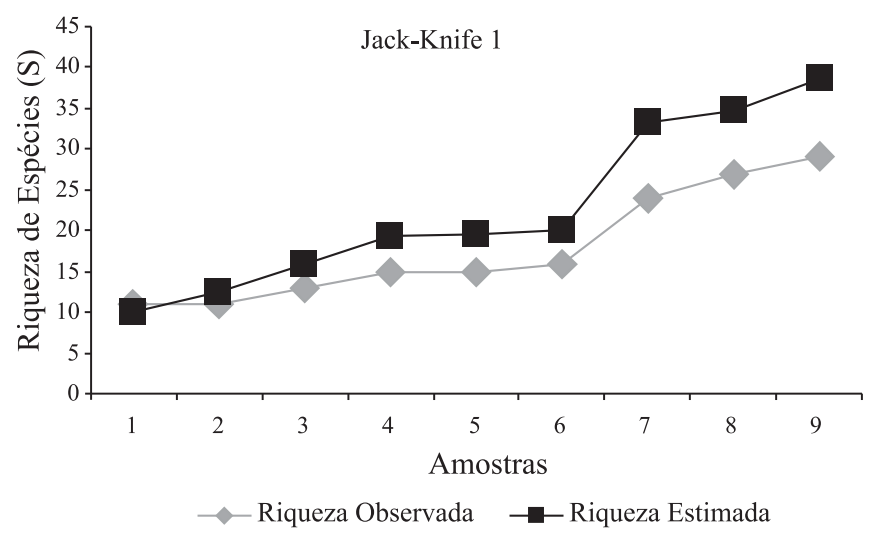

Fig. 4. Comparação entre a riqueza de espécies obtidas e a riqueza estimada (Jack-Knife 1), para a comunidade de Formicidae associada a copa de A. phalerata Mart. (Arecaceae), durante o período de cheia no Pantanal de Poconé, Mato Grosso. 

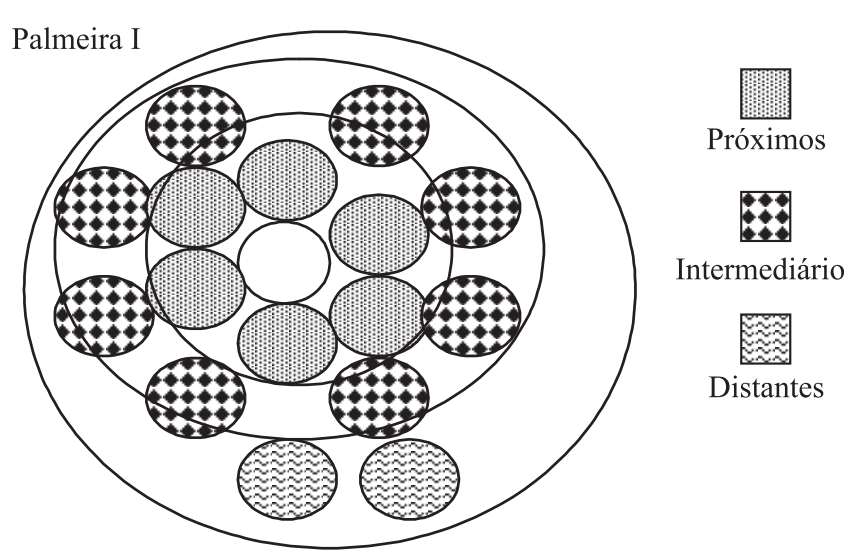

Intermediários
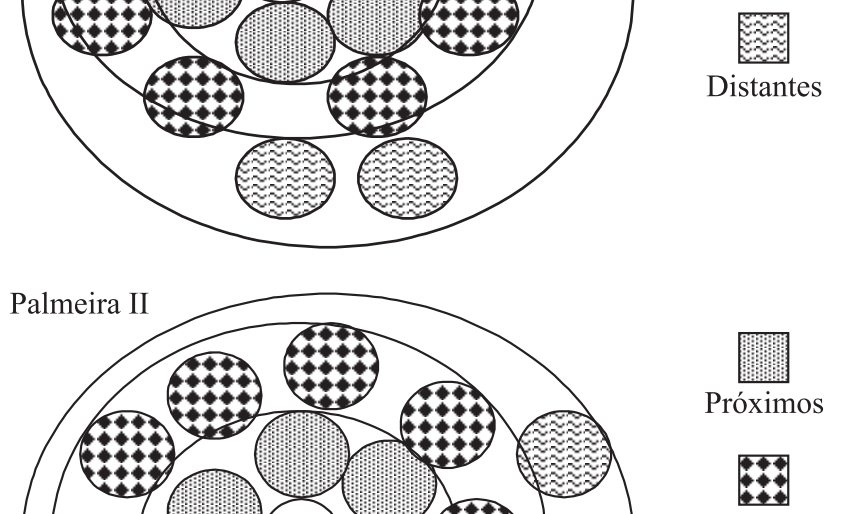

Intermediários
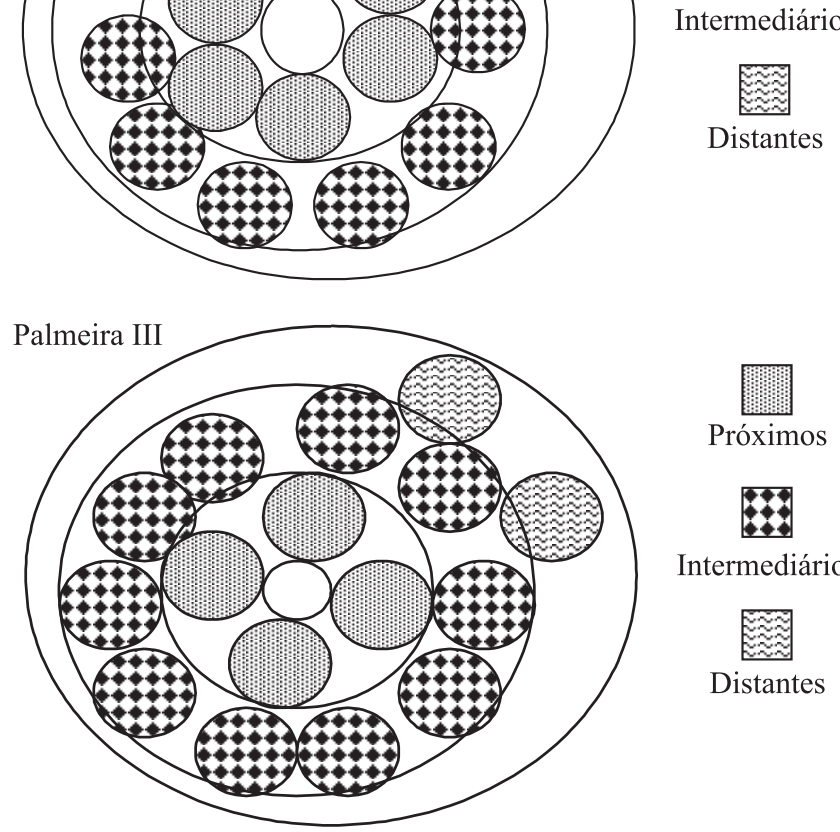

Intermediários

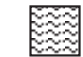

Distantes

Fig. 5. Disposição dos funis coletores debaixo de três copas de $A$. phalerata Mart. (Arecaceae) para amostragem da comunidade de Formicidae.

também verificada em relação às espécies predominantes (Tabela V). Na palmeira I a espécie dominante foi Crematogaster (Orthocrema) sp.1 pr. quadriformis (86 indivíduos; 36,0\%), seguida por Camponotus (Myrmobrachys) crassus (71 indivíduos; 29,7\%). A palmeira II apresentou a menor abundância com apenas 58 indivíduos e predominância de Pheidole sp. 1 (18 indivíduos; 31,0\%) e Camponotus (Myrmobrachys) crassus (13 indivíduos; 24,0\%). Pheidole sp. 2 (284 indivíduos; 42,4\%) e Brachymyrmex sp. (119 indivíduos; $17,8 \%$ ), foram mais representativas na palmeira III.

O gênero Camponotus (5 spp.) possui indivíduos generalistas com algumas espécies predadoras potenciais que vivem sobre árvores frutíferas em bosques, muitas vezes utilizadas como agentes de controle biológico (Cuezzo 1998). Floren \& Linsenmair (1998) citaram esse gênero como dominante em copas nos estudos realizados na Malásia. Alguns grupos podem utilizar as plantas como local de nidificação, aproveitando ramos, caules escavados por coleópteros ou outros insetos, raízes de epífitas, entre outros, a exemplo de Strumigenys Smith 1860 e Hypoponera Santschi 1938, que usam o sistema radicular de epífitas tropicais e bulbos de orquídeas para construir seus ninhos (Cuezzo 1998).

Wilson (1987), analisando amostras realizadas no Peru, identificou 40 gêneros e 135 espécies de formigas, representando também o grupo dominante nestes estudos. Dos 17 gêneros encontrados em copas de A. phalerata, 12 são comuns àqueles amostrados por Wilson (1987), podendose citar Odontomachus Latreille, 1804 (Ponerinae), Pseudomyrmex Lund, 1831 (Pseudomyrmecinae), Pheidole Westwood, 1841, Solenopsis Westwood, 1841, Crematogaster Lund, 1831, Cephalotes Latreille, 1802, Atta Fabrícius, 1804, Strumigenys Smith, 1860 (Myrmicinae), Brachymyrmex Mayr, 1868, Paratrechina Motschulsky, 1863 e Camponotus Mayr, 1861 (Formicinae), além de Labidus praedator (Fr. Smith, 1858) (Ecitoninae).

No Pantanal mato-grossense, Santos et al. (2003) obtiveram um total de 3197 formigas, representando $18,7 \%$ do total de artrópodes amostrados durante a fase terrestre (seca) em seis copas de A. phalerata, sendo esse grupo um dos dominantes na composição da artropodofauna durante esse período. Resultados similares foram obtidos por Marques et al. (2001), que apresentam Formicidae como dominante em copas de Vochysia divergens Pohl (Vochysiaceae) nessa mesma região.

A dominância de Formicidae sobre outros grupos de artrópodes foi verificada nos estudos de Adis et al. (1984) e Erwin (1983), em diferentes tipos de florestas na Amazônia Central, correspondendo a $43 \%$ e $51 \%$ do total de organismos coletados, respectivamente. Ainda na Amazônia, Harada \& Adis $(1997,1998)$ avaliaram copas de Calophyllum brasiliense Camb. (Guttiferae) e Goupia glabra Aublet. (Celastraceae) e obtiveram resultados similares. O mesmo ocorreu em estudos realizados em Borneo (Stork 1991) e na Malásia, em que os Formicidae corresponderam a 54\% dos artrópodes obtidos (Floren \& Linsenmair 1997, 1998).

Estudos na Amazônia Central (Adis 1997; Majer \& Delabie 1994) e Pantanal mato-grossense (Adis et al. 2001),

Tabela I. Dados de altura, DAP, diâmetro da copa das três palmeiras de A. phalerata Mart. (Arecaceae), amostradas durante o período de cheia no Pantanal de Poconé, Mato Grosso.

\begin{tabular}{ccccc}
\hline Palmeira & $\begin{array}{c}\text { Altura } \\
(\mathrm{m})\end{array}$ & $\begin{array}{c}\text { DAP } \\
(\mathrm{m})\end{array}$ & $\begin{array}{c}\text { Diâmetro da } \\
\text { copa }\end{array}$ & $\begin{array}{c}\text { Funis } \\
\text { Coletores }\end{array}$ \\
\hline I & 2,77 & 2,75 & 7,82 & 16 \\
II & 2,53 & 2,35 & 7,31 & 17 \\
III & 3,79 & 1,43 & 6,89 & 16 \\
\hline
\end{tabular}


Tabela II. Táxons de Formicidae obtidos em três copas de A. phalerata Mart. (Arecaceae), durante o período de cheia no Pantanal de Poconé, Mato Grosso, distribuídos entre as três coletas realizadas em cada uma das palmeiras avaliadas.

\begin{tabular}{|c|c|c|c|c|c|c|c|c|c|c|c|c|}
\hline \multirow{2}{*}{\multicolumn{3}{|c|}{ Táxons }} & \multicolumn{9}{|c|}{ Palmeiras } & \multirow{3}{*}{$\Sigma$} \\
\hline & & & \multicolumn{3}{|c|}{ I } & & \multicolumn{3}{|c|}{ III } & \\
\hline Subfamílias & Tribos & Espécies & $* 1^{\mathrm{a}}$ & $2^{\mathrm{a}}$ & $3^{\mathrm{a}}$ & $1^{\mathrm{a}}$ & $2^{\mathrm{a}}$ & $3^{\mathrm{a}}$ & $1^{\mathrm{a}}$ & $2^{\mathrm{a}}$ & $3^{\mathrm{a}}$ & \\
\hline \multirow[t]{15}{*}{ Myrmicinae } & Cephalotini & Cephalotes pallidus De Andrade, 1999 & 1 & - & - & - & - & - & - & 1 & - & 2 \\
\hline & & Cephalotes clypeatus (Fabricius, 1804) & - & - & - & - & - & - & 1 & - & - & 1 \\
\hline & Attini & Mycocepurus goeldii Forel, 1893 & - & - & - & - & - & - & - & - & 1 & 1 \\
\hline & & Atta sp. & - & - & - & - & - & 2 & - & - & - & 2 \\
\hline & Crematogastrini & Crematogaster (Orthocrema) sp. 1 pr. & & & & & & & & & & \\
\hline & & quadriformis & 79 & 16 & 1 & - & - & 1 & 2 & 1 & 1 & 101 \\
\hline & Solenopsidini & Solenopsis (Diplorhoptrum) sp.1 & 8 & 10 & 2 & 2 & 1 & - & 13 & 11 & - & 47 \\
\hline & & Solenopsis (Diplorhoptrum) sp.2 & 3 & 2 & - & 1 & - & - & 29 & 9 & - & 44 \\
\hline & & Solenopsis (Diplorhoptrum) sp.3 & - & - & - & - & - & - & - & 2 & - & 2 \\
\hline & & Solenopsis (Diplorhoptrum) sp.4 & - & - & - & - & - & - & 1 & - & - & 1 \\
\hline & & Solenopsis saevissima (Fr. Smith, 1855) & - & - & 15 & - & - & 7 & 1 & - & - & 23 \\
\hline & Pheidolini & Pheidole sp.1 & 1 & - & 9 & 2 & - & 17 & 5 & - & 10 & 44 \\
\hline & & Pheidole sp. 2 & - & - & - & - & - & - & 248 & 36 & - & 284 \\
\hline & Dacetini & Strumigenys elongata Roger, 1863 & - & - & - & - & - & - & 4 & 1 & - & 5 \\
\hline & & Pyramica sp. & - & - & - & 1 & - & - & - & - & - & 1 \\
\hline \multirow[t]{12}{*}{ Formicinae } & Lasiini & Paratrechina sp. & - & - & 3 & - & - & 2 & - & - & 5 & 10 \\
\hline & Brachymyrmecini & Brachymyrmex sp. & 2 & - & - & 6 & 1 & - & 98 & 12 & - & 119 \\
\hline & Camponotini & Camponotus (Myrmobrachys) crassus & & & & & & & & & & \\
\hline & & Mayr, 1862 & 69 & 1 & 1 & 13 & - & - & 95 & 3 & - & 182 \\
\hline & & Camponotus (Myrmobrachys) sp.1 & 4 & - & - & - & - & - & 1 & - & - & 5 \\
\hline & & Camponotus (Myrmobrachys) sp.2 & - & - & - & - & - & - & 8 & 1 & - & 9 \\
\hline & & Camponotus (Myrmosphincta) & & & & & & & & & & \\
\hline & & sexguttatus (Fabrícius, 1793) & - & - & - & 2 & - & - & 26 & 1 & - & 29 \\
\hline & & Camponotus (Tanaemyrmex) & & & & & & & & & & \\
\hline & & melanoticus Emery, 1894 & 2 & - & - & - & - & - & - & - & - & 2 \\
\hline & & Camponotus (Myrmotryx) rufipes & & & & & & & & & & \\
\hline & & (Fabrícius, 1775) & 1 & 5 & - & - & - & - & 17 & - & 1 & 24 \\
\hline \multirow[t]{3}{*}{ Ponerinae } & Odontomachini & Odontomachus bauri Emery, 1891 & - & - & - & - & - & - & 17 & 1 & - & 18 \\
\hline & Ponerini & Hypoponera sp.1 & - & - & - & - & - & - & 1 & - & - & 1 \\
\hline & & Hуроропега sp.2 & - & - & - & - & - & - & 1 & - & - & 1 \\
\hline \multirow[t]{2}{*}{ Ecitoninae } & Ecitonini & Labidus praedator (Fr. Smith, 1858) & - & - & - & - & - & - & - & - & 1 & 1 \\
\hline & & Neivamyrmex pilosus (Fr. Smith, 1858) & - & - & - & - & - & - & - & 1 & - & 1 \\
\hline Dolichoderinae & Tapinomini & Linepithema sp. & 2 & - & 2 & - & - & - & - & - & - & 4 \\
\hline $\begin{array}{l}\text { Pseudomyrme- } \\
\text { cinae }\end{array}$ & & Pseudomyrmex gracilis (Fabrícius, 1804 & - & - & - & - & - & - & 1 & 1 & - & 2 \\
\hline Total & & & 172 & 34 & 33 & 27 & 2 & 29 & 569 & 82 & 19 & 966 \\
\hline$(\%)$ & & & 17,8 & 3,5 & 3,4 & 2,8 & 0,2 & 3,0 & 58,9 & 8,5 & 1,9 & 100 \\
\hline
\end{tabular}

* - Número da coleta realizada em cada uma das palmeiras.

demonstraram que as formigas representam um importante componente da artropodofauna das áreas alagáveis, pois participam de grande parte dos processos ecológicos apresentando diferentes estratégias de sobrevivência e comportamentos sociais nessas áreas.

O índice de Shannon-Wiener (H') apontou baixa diversidade $(2,185)$ e equitabilidade média $(0,649)$, indicando que a distribuição da abundância dos indivíduos não foi homogênea. Entretanto, o índice de Simpson (D) demonstrou uma diversidade elevada para a mesma comunidade $(0,835) \mathrm{e}$ baixa equitabilidade $(0,248)$ (Tabela VI). Tais diferenças podem estar associadas ao fato desses índices serem diferentemente influenciados pela presença de espécies dominantes ou raras.

O estimador Chao 1 evidenciou que apenas $50,8 \%$ das espécies foi amostrada, indicando que 57 espécies deveriam ter sido coletadas, ao contrario das 29 obtidas (Fig. 3), já através do estimador Jack-Knife 1, verifica-se que $74,9 \%$ da riqueza de espécies foi amostrada e que apenas 9 espécies não foram coletadas (Fig. 4). Apesar do baixo número de palmeiras avaliadas, a amostragem foi considerável, atendendo aos 
Tabela III. Índice de Similaridade de Sørenson aplicado a comunidade de Formicidae obtida em três copas de A. phalerata Mar. (Arecaceae), durante o período de cheia no Pantanal de Poconé, Mato Grosso.

\begin{tabular}{cccc}
\hline A. phalerata & I & II & III \\
\hline I & - & 0,66 & 0,57 \\
II & 0,66 & - & 0,50 \\
III & 0,57 & 0,50 & - \\
\hline
\end{tabular}

objetivos propostos, entretanto, estudos mais detalhados enfocando outras variáveis são necessários para uma melhor avaliação dessa comunidade.

Habitat. De acordo com a análise do habitat utilizado pelas espécies associadas a copa de $A$. phalerata, verifica-se que a maioria (14 spp.; 48,2\%) é representada por espécies que nidificam principalmente no solo, ou seja, aquelas que vivem

Tabela IV. Indivíduos de Formicidae obtidos durante a primeira e segunda coletas, em três copas de $A$. phalerata Mart. (Arecaceae), durante o período de cheia no Pantanal de Poconé, Mato Grosso, distribuídos de acordo com o posicionamento dos funis coletores.

\begin{tabular}{|c|c|c|c|c|c|c|c|c|c|c|c|c|c|}
\hline \multicolumn{3}{|l|}{ Táxons } & \multicolumn{3}{|c|}{ A. phalerata } & \multicolumn{3}{|c|}{$\begin{array}{l}\text { A. phalerata } \\
\text { II }\end{array}$} & \multicolumn{3}{|c|}{$\begin{array}{l}\text { A. phalerata } \\
\text { III }\end{array}$} & & \multirow[t]{2}{*}{$\begin{array}{c}\text { Ind./I } \\
\mathrm{m}^{2} \\
\end{array}$} \\
\hline Subfamílias & Tribos & Espécies & $\mathrm{P}^{*}$ & I & $\mathrm{D}$ & $\mathrm{P}$ & I & $\mathrm{D}$ & $\mathrm{P}$ & I & $\mathrm{D}$ & & \\
\hline \multirow[t]{14}{*}{ Myrmicinae } & Cephalotini & Cephalotes pallidus De Andrade, 1999 & 1 & - & - & - & - & - & - & 1 & - & 2 & $<0,1$ \\
\hline & & Cephalotes clypeatus (Fabricius, 1804) & - & - & - & - & - & - & 1 & - & - & 1 & $<0,1$ \\
\hline & Attini & Myсосеритиs goeldii Forel, 1893 ** & - & - & - & - & - & - & - & - & - & 0 & - \\
\hline & & Atta sp. ** & - & - & - & - & - & - & - & - & - & 0 & - \\
\hline & Crematogastrini & $\begin{array}{l}\text { Crematogaster (Orthocrema) sp. } 1 \text { pr. } \\
\text { quadriformis }\end{array}$ & 89 & 6 & - & - & - & - & - & 2 & 1 & 98 & 2,0 \\
\hline & Solenopsidini & Solenopsis (Diplorhoptrum) sp.1 & 17 & 1 & - & 2 & 1 & - & 20 & 3 & 1 & 45 & 0,9 \\
\hline & & Solenopsis (Diplorhoptrum) sp.2 & 5 & - & - & - & 1 & - & 37 & 1 & - & 44 & 0,9 \\
\hline & & Solenopsis (Diplorhoptrum) sp.3 & - & - & - & - & - & - & 2 & - & - & 2 & $<0,1$ \\
\hline & & Solenopsis (Diplorhoptrum) sp.4 & - & - & - & - & - & - & 1 & - & - & 1 & $<0,1$ \\
\hline & & Solenopsis saevissima (Fr. Smith, 1855) & - & - & - & - & - & - & - & 1 & - & 1 & $<0,1$ \\
\hline & Pheidolini & Pheidole sp.1 & 1 & - & - & 2 & - & - & 3 & 2 & - & 8 & 0,1 \\
\hline & & Pheidole sp.2 & - & - & - & - & - & - & 274 & 9 & 1 & 284 & 5,8 \\
\hline & Dacetini & Strumigenys elongata Roger, 1863 & - & - & - & - & - & - & 4 & 1 & - & 5 & 0,1 \\
\hline & & Pyramica sp. & - & - & - & - & 1 & - & - & - & - & 1 & $<0,1$ \\
\hline \multirow[t]{10}{*}{ Formicinae } & Lasiini & Paratrechina sp. $* *$ & - & - & - & - & - & - & - & - & - & 0 & - \\
\hline & Brachymyrmecini & Brachymyrmex sp. & 2 & - & - & 4 & 2 & 1 & 108 & 2 & - & 119 & 2,4 \\
\hline & Camponotini & Camponotus (Myrmobrachys) crassus & & & & & & & & & & & \\
\hline & & Mayr, 1862 & 65 & 4 & 1 & 10 & 3 & - & 95 & 2 & 1 & 181 & 3,7 \\
\hline & & Camponotus (Myrmobrachys) sp.1 & 4 & - & - & - & - & - & 1 & - & - & 5 & 0,1 \\
\hline & & Camponotus (Myrmobrachys) sp.2 & - & - & - & - & - & - & 6 & 3 & - & 9 & 0,2 \\
\hline & & $\begin{array}{l}\text { Camponotus (Myrmosphincta) } \\
\text { sexguttatus (Fabrícius, 1793) }\end{array}$ & - & - & - & 2 & - & - & 15 & 9 & 3 & 29 & 0,6 \\
\hline & & Camponotus (Tanaemyrmex) & & & & & & & & & & & \\
\hline & & melanoticus Emery, 1894 & 2 & - & - & - & - & - & - & - & - & 2 & $<0,1$ \\
\hline & & $\begin{array}{l}\text { Camponotus (Myrmotryx) rufipes } \\
\text { (Fabrícius, 1775) }\end{array}$ & 6 & - & - & - & - & - & 16 & 1 & - & 23 & 0,4 \\
\hline \multirow[t]{3}{*}{ Ponerinae } & Odont & Odontomachus bauri Emery, 1891 & - & - & - & - & - & - & 16 & 2 & - & 18 & 0,3 \\
\hline & Ponerini & Hypoponera sp.1 & - & - & - & - & - & - & - & 1 & - & 1 & $<0,1$ \\
\hline & & Hypoponera sp.2 & - & - & - & - & - & - & - & 1 & - & 1 & $<0,1$ \\
\hline \multirow[t]{2}{*}{ Ecitoninae } & Ecitonini & Labidus praedator (Fr. Smit) & - & - & - & - & - & - & - & - & - & 0 & - \\
\hline & & ilosus (Fr. Smith, 1858) & - & - & - & - & - & - & - & 1 & - & 1 & $<0,1$ \\
\hline $\begin{array}{l}\text { Dolichode- } \\
\text { rinae }\end{array}$ & Tapinomini & Linepithema sp. & 1 & 1 & - & - & - & - & - & - & - & 2 & $<0,1$ \\
\hline $\begin{array}{l}\text { Pseudomyr- } \\
\text { mecinae }\end{array}$ & & $\begin{array}{l}\text { Pseudomyrmex gracilis (Fabrícius, } \\
\text { 1804) }\end{array}$ & - & - & - & - & - & - & 1 & 1 & - & 2 & $<0,1$ \\
\hline Total & & & 193 & 12 & 1 & 20 & 8 & 1 & 600 & 43 & 7 & 885 & 18,0 \\
\hline$(\%)$ & & & 21,8 & 1,3 & 0,1 & 2,3 & 0,9 & 0,1 & 67,8 & 4,9 & 0,8 & 100 & \\
\hline
\end{tabular}

*- Posicionamento dos funis coletores em relação ao caule da palmeira $(\mathrm{P}=$ próximo; $\mathrm{I}=$ intermediário e $\mathrm{D}=$ distante $)$

** - Táxons amostrados somente na terceira coleta 
Tabela V. Táxons, número de indivíduos (N), proporção (\%), hábito alimentar e habitat de Formicidae, obtidos em copas de $A$. phalerata Mart. (Arecaceae), durante o período de cheia no Pantanal de Poconé, Mato Grosso.

\begin{tabular}{|c|c|c|c|c|c|}
\hline \multirow[t]{2}{*}{ Táxons } & \multirow[t]{2}{*}{ Habitat } & \multirow[t]{2}{*}{$\begin{array}{c}\text { Hábito } \\
\text { Alimentar }\end{array}$} & \multicolumn{2}{|c|}{ Abundância } & \multirow{2}{*}{$\begin{array}{c}\text { Freqüência } \\
(\%)\end{array}$} \\
\hline & & & $\mathrm{N}$ & $(\%)$ & \\
\hline \multicolumn{6}{|l|}{ Myrmicinae/Cephalotini } \\
\hline Cephalotes pallidus De Andrade, 1999 & Arborícola & Líquidos e Pólen & 2 & 0,2 & 66,6 \\
\hline Cephalotes clypeatus (Fabricius, 1804) & Arborícola & Líquidos e Pólen & 1 & 0,1 & 33,3 \\
\hline \multicolumn{6}{|l|}{ Myrmicinae/Attini } \\
\hline Mycocepurus goeldii Forel, 1893 & Terrícola & Fungívoro & 1 & 0,1 & 33,3 \\
\hline $\begin{array}{l}\text { Atta sp. } \\
\text { Myrmicinae/Crematogastrini }\end{array}$ & Terrícola & Fungívoro & 2 & 0,2 & 33,3 \\
\hline Myrmicinae/Solenopsidini & ? Arborícola & Onívoro & 101 & 10,5 & 100,0 \\
\hline Solenopsis (Diplorhoptrum) sp. 1 & ? Terrícola & Onívoro & 47 & 4,9 & 100,0 \\
\hline Solenopsis (Diplorhoptrum) sp. 2 & ? Terrícola & Onívoro & 44 & 4,6 & 100,0 \\
\hline Solenopsis (Diplorhoptrum) sp. 3 & ? Terrícola & Onívoro & 2 & 0,2 & 33,3 \\
\hline Solenopsis (Diplorhoptrum) sp. 4 & ? Terrícola & Onívoro & 1 & 0,1 & 33,3 \\
\hline Solenopsis saevissima (Fr. Smith, 1855) & Terrícola & Onívoro & 23 & 2,4 & 100,0 \\
\hline \multicolumn{6}{|l|}{ Myrmicinae/Pheidolini } \\
\hline Pheidole sp. 1 & ? Terrícola & Onívoro & 44 & 4,6 & 100,0 \\
\hline Pheidole sp. 2 & ? Terrícola & Onívoro & 284 & 29,4 & 33,3 \\
\hline \multicolumn{6}{|l|}{ Myrmicinae/Dacetini } \\
\hline Strumigenys elongata Roger, 1863 & ? Terrícola & Predador & 5 & 0,5 & 33,3 \\
\hline Pyramica sp. & ? Terrícola & Predador & 1 & 0,1 & 33,3 \\
\hline \multicolumn{6}{|l|}{ Formicinae/Lasiini } \\
\hline \multirow{2}{*}{\multicolumn{6}{|c|}{ Formicinae/Brachymyrmecini }} \\
\hline & & & & & \\
\hline \multirow{2}{*}{\multicolumn{6}{|c|}{ Formicinae/Camponotini }} \\
\hline & & & & & \\
\hline Camponotus (Myrmobrachys) crassus Mayr, 1862 & ? Arborícola & Onívoro & 182 & 18,8 & 100,0 \\
\hline Camponotus (Myrmobrachys) sp. 1 & ? Arborícola & Onívoro & 5 & 0,5 & 66,6 \\
\hline Camponotus (Myrmobrachys) sp. 2 & ?Arborícola & Onívoro & 9 & 0,9 & 33,3 \\
\hline Camponotus (Myrmosphincta) sexguttatus (Fabricius 1793) & ? Arborícola & Onívoro & 29 & 3,0 & 100,0 \\
\hline Camponotus (Tanaemyrmex) melanoticus Emery, 1891 & ? Arborícola & Onívoro & 2 & 0,2 & 33,3 \\
\hline Camponotus (Myrmotryx) rufipes (Fabricius, 1775) & ? Terrícola & Onívoro & 24 & 2,5 & 66,6 \\
\hline \multicolumn{6}{|l|}{ Ponerinae/Odontomachini } \\
\hline Odontomachus bauri Emery, 1891 & ? Terrícola & Predador & 18 & 1,9 & 33,3 \\
\hline \multicolumn{6}{|l|}{ Ponerinae/Ponerini } \\
\hline Hypoponera sp. 1 & ? Terrícola & Predador & 1 & 0,1 & 33,3 \\
\hline Hypoponera sp. 2 & ? Terrícola & Predador & 1 & 0,1 & 33,3 \\
\hline \multicolumn{6}{|l|}{ Ecitoninae/Ecitonini } \\
\hline Neivamyrmex pilosus (Fr. Smith, 1858) & Terrícola & Predador & 1 & 0,1 & 33,3 \\
\hline Labidus praedator (Fr. Smith, 1858) & Terrícola & Predador & 1 & 0,1 & 33,3 \\
\hline \multicolumn{6}{|l|}{ Dolichoderinae/Tapinomini } \\
\hline Linepithema sp. & ? Terrícola & Líquidos e Pólen & 4 & 0,4 & 33,3 \\
\hline \multicolumn{6}{|l|}{ Pseudomyrmecinae/Pseudomyrmecini } \\
\hline Pseudomyrmex gracilis (Fabricius, 1804) & Arborícola & Predador & 2 & 0,2 & 33,3 \\
\hline Total & & & 966 & 100,0 & \\
\hline
\end{tabular}

geralmente associadas a fragmentos em decomposição como folhas, galhos ou madeira caída. Além disso, 5 espécies (17,2\%) são consideradas típicas de solo (Tabela V).

A provável explicação para a ocorrência de várias espécies habitantes de solo em copas de A. phalerata, pode estar associada ao desenvolvimento de estratégias de sobrevivência durante as inundações periódicas no Pantanal mato-grossense como as migrações do solo em direção à copa.

Segundo Way \& Bolton (1997) diversas espécies utilizam os recursos oferecidos pelas bases das folhas de palmeiras 
Tabela VI. Índices de diversidade para a comunidade de Formicidae em três copas de A. phalerata Mart. (Arecaceae), durante o período de cheia no Pantanal de Poconé, Mato Grosso.

\begin{tabular}{ccccc}
\hline Shannon-Wiener $\left(\mathrm{H}^{\prime}\right)$ & Equitabilidade & Simpson $(\mathrm{D})$ & Equitabilidade & Riqueza (S) \\
\hline 2,185 & 0,649 & 0,835 & 0,248 & 29 \\
\hline
\end{tabular}

para nidificar e é provável que muitas, encontradas normalmente sobre o solo, migrem para essas regiões da planta durante o período de inundação. Outro fator a ser considerado é a grande quantidade de matéria orgânica acumulada nas bainhas foliares dessa palmeira, constituindo uma espécie de solo suspenso, que oferece uma grande variedade de nichos para as formigas, principalmente aquelas que vivem em serapilheira. Battirola et al. (2004b) ressaltaram também que, além de servir como habitat, a matéria orgânica acumulada na copa de $A$. phalerata é utilizada como refugio durante as inundações periódicas por diversos grupos de artrópodes no Pantanal mato-grossense.

Todos esses fatores confirmam que as formigas de fato realizam migrações verticais no Pantanal mato-grossense como já abordado por Adis et al. (2001). Apesar disso, estudos ecológicos e biológicos mais detalhados em anos de cheias extremas no Pantanal são necessários para avaliar de forma precisa a inter-relação entre esses habitats.

Algumas espécies de Pheidole apresentam estratégias de fuga dos ninhos mediante indícios de inundação, pois as operárias respondem a estímulos desencadeados por pequenas quantidades de água presente na entrada dos seus ninhos, mobilizando rapidamente toda a colônia, conduzindo seus companheiros por galerias, por meio de feromônios (Wilson 1986). No Pantanal mato-grossense essa estratégia poderia ser desenvolvida por essas formigas para garantir a sobrevivência da colônia durante as inundações periódicas.

Apenas 3 espécies (10,3\%), Cephalotes pallidus De Andrade, 1999 C. clypeatus Fabrícius, 1804 e Pseudomyrmex gracilis Fabrícius, 1804, são tipicamente arborícolas, e 7 espécies $(24,1 \%)$, dentre elas as pertencentes ao gênero Camponotus, utilizam recursos de nidificação em árvores de forma oportunista, com um habitat incerto.

Harada \& Adis (1997), quando avaliaram a comunidade de formigas em copas de G. glabra Aublet. (Celastraceae) na Amazônia a aproximadamente $45 \mathrm{~m}$ de altura, também amostraram indivíduos pertencentes aos gêneros Camponotus (3 spp.), Pheidole (3 spp.) e Solenopsis (9 spp.), demonstrando que esses grupos podem ser terrícolas ou arborícolas.

Hábitos alimentares. $\mathrm{O}$ arranjo das espécies de formigas dentro das comunidades é influenciado pela distribuição dos recursos a serem explorados, bem como pelas estratégias utilizadas por esses organismos para a sua obtenção (Fowler et al. 1991). Dessa maneira a coexistência de diferentes espécies em um mesmo habitat, depende da amplitude ecológica de cada uma dentro da comunidade.
Neste estudo foram amostrados 4 grupos de formigas de acordo com seu hábito alimentar. As espécies onívoras foram as dominantes (916 indivíduos; 94,8\%), seguidas pelas predadoras (40 indivíduos; $4,1 \%$ ), aquelas que alimentam-se de líquidos (7 indivíduos; $0,7 \%$ ) e a menor abundância foi registrada para as fungívoras (3 indivíduos; $0,3 \%$ ) (Tabela V). A predominância de onívoras (16 spp.) em todas as palmeiras avaliadas, tanto em riqueza $(55,2 \%)$, quanto em abundância $(94,8 \%)$, caracteriza essa comunidade pela presença de espécies generalistas que podem explorar uma maior variedade de recursos no ambiente, enquanto grupos especializados como as fungívoras e aquelas que alimentam de líquidos apresentaram baixa abundância em todas as palmeiras (Tabela VII).

Dentre as onívoras dominantes nesse estudo destacam-se as espécies Pheidole sp. 2 (284 indivíduos; 29,4\%), Camponotus crassus (182 indivíduos; 18,8\%) e Crematogaster (Orthocrema) sp. 1 pr. quadriformis (101 indivíduos; 10,5\%) (Tabela V). Apesar das formigas serem consideradas predadoras por natureza, alguns grupos possuem uma maior plasticidade em explorar outros recursos alimentares dependendo das condições ambientais vigentes e da disponibilidade de recursos necessários para sustentar a colônia. Algumas espécies de Camponotus e Crematogaster coletam soluções açucaradas produzidas por nectários florais e extraflorais e por insetos principalmente homópteros e alguns lepidópteros, em certos casos, ocorrendo especificidade entre as interações (Delabie 2001; Fowler et al. 1991; Tillberg \& Breed 2004).

As espécies predadoras foram representadas principalmente por Odontomachus bauri Emery, 1891 (18 indivíduos; 1,9\%), Paratrechina sp. (10 indivíduos; 1,0\%) e Strumigenys elongata Roger, 1863 (5 indivíduos; 0,5\%) (Tabela V). As formigas predadoras nos ecossistemas naturais podem apresentar especificidade alimentar, ou hábitos generalistas

Tabela VII. Distribuição de Formicidae de acordo com seu hábito alimentar em copas de $A$. phalerata Mart. (Arecaceae), durante o período de cheia no Pantanal de Poconé, Mato Grosso.

\begin{tabular}{lcccc}
\hline \multicolumn{5}{c}{ Palmeira } \\
\hline Hábito alimentar & I & II & III & Média \\
\hline Onívoro & 97,9 & 94,9 & 95,2 & 96,0 \\
Predador & - & 1,7 & 4,4 & 2,0 \\
Fungívoro & - & 3,4 & 0,1 & 1,2 \\
Líquidos e Pólen & 2,1 & - & 0,3 & 0,8 \\
\hline Total & $100 \%$ & $100 \%$ & $100 \%$ & \\
\hline
\end{tabular}


Tabela VIII. Abundância, riqueza e densidade de Formicidae, obtida em três copas de A. phalerata Mart. (Arecaceae), no Pantanal de Poconé, Mato Grosso, de acordo com o posicionamento dos funis coletores, em cada uma das palmeiras avaliadas.

\begin{tabular}{lcccccccccccccc}
\hline Palmeiras & \multicolumn{1}{c}{ I } & \multicolumn{1}{c}{ II } & \multicolumn{1}{c}{ III } \\
\hline Posicionamento dos funis & $*$ & $\mathrm{I}$ & $\mathrm{D}$ & $\Sigma$ & $\mathrm{P}$ & $\mathrm{I}$ & $\mathrm{D}$ & $\Sigma$ & $\mathrm{P}$ & $\mathrm{I}$ & $\mathrm{D}$ & $\Sigma$ & total \\
\hline Área $\left(\mathrm{m}^{2}\right)$ & 6 & 8 & 2 & 16 & 6 & 10 & 1 & 17 & 4 & 10 & 2 & 16 & 49 \\
Abundância (n ${ }^{\circ}$ ind.) & 193 & 12 & 1 & 206 & 20 & 8 & 1 & 29 & 600 & 43 & 7 & 650 & 885 \\
Riqueza Subfamílias & 3 & 3 & 1 & 3 & 2 & 2 & 1 & 2 & 4 & 5 & 2 & 5 & 6 \\
Riqueza Tribos & 7 & 4 & 1 & 7 & 4 & 4 & 1 & 5 & 8 & 11 & 4 & 12 & 13 \\
Riqueza Espécies & 11 & 4 & 1 & 14 & 5 & 5 & 1 & 11 & 16 & 18 & 5 & 25 & 29 \\
Densidade (Indivíduos $\left./ \mathrm{m}^{2}\right)$ & 32,2 & 1,5 & 0,5 & 12,9 & 3,3 & 0,8 & 1,0 & 1,7 & 150,0 & 4,3 & 3,5 & 40,6 & 18,1 \\
\hline
\end{tabular}

* Posicionamento dos funis coletores em relação ao caule da palmeira ( $\mathrm{P}=$ próximo; $\mathrm{I}=$ intermediário e $\mathrm{D}=$ distante)

como as representantes do gênero Labidus Jurine 1807(1 indivíduo; $0,1 \%$ ), que consomem qualquer material de origem animal. Alguns grupos como Neivamyrmex Borgmeier, 1894 (1 indivíduo; $0,1 \%$ ) e Labidus (1 indivíduo;0,1\%), possuem estratégias de captura coletiva, chamadas formigas de correição, comuns sobre o solo, o que pode explicar a baixa abundância desses grupos na copa de $A$. phalerata.

Atta sp. (2 indivíduos; 0,2\%) e Mycocepurus goeldii Forel, 1893 ( 1 indivíduo; 0,1\%), foram as únicas representantes das formigas fungívoras obtidas em copas de A. phalerata. Normalmente indivíduos de Atta apresentam grandes colônias alimentadas por fungos cultivados dentro dos ninhos localizados geralmente no solo. Uma baixa abundância também foi observada para as formigas que se alimentam de substâncias líquidas, representadas por três espécies, Linepithema sp. (4 indivíduos; 0,4\%), Cephalotes pallidus ( 2 indivíduos; 0,2\%) e Cephalotes clypeatus (1 indivíduo; 0,1\%) amostradas neste estudo (Tabela V).

Distribuição espacial. O número de funis coletores variou conforme o tamanho e a arquitetura da copa analisada. Nesta pesquisa foram utilizados 16 funis nas palmeiras I e III, e 17 na palmeira II. Com base no mapeamento definiram-se como posicionamentos para os funis coletores aqueles localizados próximos ao caule $(\mathrm{P})$, os de posição intermediária (I) e os distantes do caule (D), nas bordas da copa (Fig. 5).

Foram amostradas 813 formigas $(91,9 \%)$ nos funis localizados próximos ao caule $(\mathrm{P}), 63(7,1 \%)$ nos intermediários (I) e apenas 9 indivíduos $(1,0 \%)$ naqueles distantes do caule (D) (Tabelas IV e VIII). Essa abundância obtida nos funis próximos ao caule é refletida nas densidades de indivíduos, sendo a palmeira de número III ( 4 funis $=4 \mathrm{~m}^{2}$ ) a que apresentou o maior valor $\left(150,0 \pm 97,7\right.$ indivíduos $\left./ \mathrm{m}^{2}\right)$, seguida pelas de número I e II $\left(6\right.$ funis $\left.=6 \mathrm{~m}^{2}\right)\left(32,2 \pm 26,2\right.$ indivíduos $/ \mathrm{m}^{2}$ e $3,3 \pm 2,8$ indivíduos/m2), respectivamente. Com relação ao número de subfamílias, tribos e espécies, esses funis também tiveram os maiores valores, com exceção da palmeira III que apresentou maior riqueza de subfamílias, tribos e espécies nos funis intermediários (I). Essa diferença pode estar associada a menor área amostral próxima ao caule presente nessa palmeira (Tabelas IV e VIII).

Apesar do baixo número de funis posicionados distantes dos caules, a riqueza de espécies foi considerável, principalmente na palmeira III. Entretanto, nas demais palmeiras os funis distantes amostraram apenas um indivíduo cada, demonstrando que a maior parte das espécies que compõem a comunidade utiliza a região central da copa como habitat, provavelmente, devido a maior disponibilidade de recursos nesse local (Tabela IV e VII).

Battirola et al. (2004b) avaliando comunidade de Araneae sobre essas mesmas palmeiras observou um padrão de distribuição similar, em que a maior abundância foi registrada nos funis próximos ao caule $(47,7 \%)$; porém a freqüência também foi elevada nos funis intermediários $(44,1 \%)$, diferentemente do padrão observado para Formicidae que apresentou maior predomínio nos funis próximos ao caule $(91,9 \%)$.

A palmeira III destaca-se em relação à abundância e diversidade da comunidade de Formicidae, pois das 29 espécies amostradas, 20 foram mais abundantes ou ocorreram somente nesta palmeira. Essa maior abundância pode estar associada a maior quantidade de matéria orgânica acumulada nas bainhas foliares dessa palmeira em relação as demais.

Vários autores salientam que a matéria orgânica acumulada nas copas constituem importante habitat para diversos grupos de artrópodes como os Formicidae, principalmente, pela diversidade de nichos disponibilizados (Delabie 2003; Kitching et al. 1997; Longino \& Nadkarni 1990; Nadkarni 1994; Nadkarni \& Longino 1990; Santos et al. 2003; Watanabe 1997).

Samways (1994) salientou que os microhabitats existentes em uma planta podem acarretar mudanças microclimáticas em suas diferentes partes e que essas diferenças proporcionam o desenvolvimento de populações que se adaptam a tais condições.

Longino \& Nadkarni (1990) demonstraram que o acúmulo de matéria orgânica nas copas de florestas tropicais propicia uma estrutura tridimensional a esse ambiente, ofertando nichos que podem ser ocupados por formigas. Erwin (2001) salientou que esses nichos contribuem para o aumento da diversidade de espécies associadas a esse ambiente, além de ser base para a estruturação das comunidades de artrópodes, afetando principalmente a composição de guildas (Lawton 1983; Samways 1994).

Por serem monocotiledôneas, as palmeiras oferecem um 
habitat aos insetos distinto daqueles encontrados nas dicotiledôneas, disponibilizando uma menor superfície foliar, porém oferecendo uma maior uniformidade, além de substratos menos complexos como as epífitas localizadas em seus galhos e bainhas que, de certa forma, incrementam o número de microhabitats disponíveis (Lucky et al. 2002). A liteira retida por palmeiras acaulescentes na Amazônia aparentemente contribuiu para o aumento da abundância e riqueza de macroinvertebrados quando comparada ao solo nos arredores (Vasconcelos 1990).

\section{CONCLUSÕES}

A comunidade de Formicidae em copas de A.phalerata mostrou-se heterogênea quanto à sua composição taxonômica e frequência de grupos. Esse fato pode estar correlacionado às diferentes condições oferecidas pela palmeira para a utilização das copas como habitat, principalmente pela diversidade de nichos disponíveis e acúmulo de matéria orgânica em suas bainhas.

O acúmulo de matéria orgânica nas bainhas dessa espécie vegetal é um fator predominante para a estruturação da comunidade de Formicidae, fato demonstrado pela maior abundância e riqueza de espécies verificadas na palmeira III, que apresentava maior quantidade de matéria orgânica em suas bainhas e folíolos.

A avaliação da distribuição das espécies de Formicidae na copa, por meio do posicionamento dos funis coletores, também comprova o papel exercido pela matéria orgânica acumulada nas bainhas foliares aderidas ao caule, bem como a maior disponibilidade de nichos na região central da copa de $A$. phalerata.

Os resultados obtidos nessa pesquisa, somados a outros formam base para futuros monitoramentos e avaliações do comportamento da comunidade de Formicidae com relação a composição e distribuição desses indivíduos nesse habitat. Além disso, A. phalerata pode ser caracterizada como uma espécie-chave nas planícies alagáveis, pois é utilizada por várias espécies de Formicidae como habitat e, provavelmente, refúgio durante as inundações periódicas nessas áreas.

Agradecimentos. Este estudo é parte dos resultados da cooperação científica entre o Instituto Max-Planck para Limnologia, Plön, Alemanha e Universidade Federal de Mato Grosso - Cuiabá, como parte do programa SHIFT (Studies of Human Impact on Forests and Floodplains in the Tropics), financiado pelo Bundesministerium für Bildung, Wissenschaft Forschung und Technologie (BMBF), Conselho Nacional de Desenvolvimento Cientifico e Tecnológico ( $\mathrm{CNPq}$ ) e Instituto Brasileiro de Meio Ambiente e Recursos Naturais Renováveis (IBAMA). Agradecemos ao técnico Francisco de Assis Rondon (UFMT), aos alunos do Laboratório de Entomologia (21A) do Instituto de Biociências da UFMT pelo auxílio nas coletas de campo, e à M.Sc. Geane Brizzola dos Santos pela leitura crítica do manuscrito e ao Programa de Pós-Graduação em Ecologia e Conservação da Biodiversidade do Instituto de Biociências da UFMT.

\section{REFERÊNCIAS}

Adis, J. 1997. Terrestrial invertebrates: survival strategies, group spectrum, dominance and activity patterns, p. 299-317. In: W. J. Junk (ed.). The Central Amazon Floodplain. Ecological Studies. Berlin,Springer-Verlag, xxiii +525 p.

Adis, J. \& H. R. O. Schubart. 1984. Ecological research on arthropods in Central Amazonian forest ecosystems with recomendations for study procedures, p. 111-144. In: J. H. Cooley \& F. B. Golley (eds.). Trends in Ecological Research for the 1980s. Nato Conference Series I: Ecology. New York, Plenun Press, 344p.

Adis, J.; Y. D. Lubin \& G. G. Montgomery. 1984. Arthropods from the canopy of inundated and Terra Firme forests near Manaus, Brazil, with critical considerations on the pyrethrum-fogging technique. Studies on Neotropical Fauna and Environment 19: 223236.

Adis, J.; M. I. Marques \& K. M. Wantzen. 2001. First observations on the survival strategies of terricolous arthropods in the northern Pantanal wetland of Brazil. Andrias 15: 127-128.

Adis, J.; Y. Basset; A. Floren; W. Hammond \& K. E. Linsenmair. 1998a. Canopy fogging of an overstory tree - recommendations for standardization. Ecotropica 4: 93-97.

Adis, J.; A. Y. Harada; C. R. V. Fonseca; W. Paarmann \& J. A. Rafael. 1998b. Arthropods obtained from the Amazonian tree species "Cupiuba" (Goupia glabra) by repeated canopy fogging with natural pyrethrum. Acta Amazônica 28: 273-283.

Battirola, L. D.; G. B. Santos; M. I. Marques \& J. Adis. 2004a. Arthropods from the canopy of Attalea phalerata Mart. (Arecaceae) in the Pantanal of Mato Grosso, Brazil. What's up? ICAN International Canopy Network. 10: 2-3.

Battirola, L. D.; M. I. Marques; J. Adis \& A. D. Brescovit. 2004b. Aspectos ecológicos da comunidade de Araneae (Arthropoda, Arachnida) em copas da palmeira Attalea phalerata Mart. (Arecaceae) no Pantanal de Poconé, Mato Grosso, Brasil. Revista Brasileira de Entomologia 48: 421-430.

Cuezzo, F. 1998. Formicidae, p. 452 - 462. In: J. J. Morrone \& S. Coscarón. Biodiversidad de Artropodos Argentinos. La Plata, Ediciones SUR, $599 \mathrm{p}$

Davidson, D. W. 1997. The role of resource imbalances in the evolutionary ecology of tropical arboreal ants. Biological Journal of the Linnean Society 61: 153-181.

Delabie, J. H. C. 2001. Trophobiosis between Formicidae and Hemiptera (Sternorrhyncha and Auchenorrhyncha): an overview. Neotropical Entomology 30: 501-516.

Delabie, J. H. C. 2003. Comunidades de formigas das árvores nas formações florestais da América do Sul, com ênfase no sudeste da Bahia. Anais do XVI Simpósio de Mirmecologia 1:109-114. UFSC, Florianópolis, Universidade Federal de Santa Catarina.

Erwin, T. L. 1982. Tropical forests: Their richness in Coleoptera and other arthropod species. The Coleopterists Bulletin 36: 74-75.

Erwin, T. L. 1983. Beetles and other insects of tropical forest canopies at Manaus, Brazil, sampled by insecticidal fogging, p. 59-75. In: S. L Sutton; T. C. Whitmore \& A. C. Chadwick (eds.). Tropical rainforest: ecology and management. Oxford, Blackwell Scientific Publications, $498 \mathrm{p}$.

Erwin, T. L. 1989. Canopy arthropod biodiversity: a chronology of sampling techniques and results. Revista Peruana de Entomologia 32: 71-77.

Erwin, T. L. 2001. Forest canopies, animal diversity, p. 19-25. In: S. A. Levin (ed.). Encyclopedia of Biodiversity. Vol. (3). San Diego, Academic Press, 870 p.

Erwin, T. L. \& J. Adis. 1982. Amazonian inundation forest their role as short-term refuges and generators of species richness and taxon pulses, p. 358-371. In: G. Prance (ed.). Biological diversification in the tropics. New York, Columbia University Press, 714p.

Fittkau, E. A. \& H. Klinge. 1973. The biomass and throphic structure of the Central Amazonian rain forest ecosystem. Biotropica 5: 2 14.

Floren, A. \& K. E. Linsenmair. 1997. Diversity and recolonization dynamics of selected arthropod groups on different tree species in a lowland rainforest in Sabah, Malaysia, with special reference to Formicidae, p. 344-381. In: N. E. Stork; J. Adis \& R. K. Didham (eds.). Canopy Arthropods. London, Chapman \& Hall, 567 p. 
Floren, A. \& K. E. Linsenmair. 1998. Diversity and recolonization of arboreal Formicidae and Coleoptera in a lowland rain forest in Sabah, Malaysia. Selbyana 19: 155-161.

Floren, A. \& K. E. Linsenmair. 2000. Do ants mosaics exist in pristine lowland rain forests? Oecologia 123: 129-137

Floren, A.; A. Biun \& Linsenmair. 2002. Arboreal ants as key predators in tropical lowland forest trees. Oecologia 131: 137-144

Fowler, H. G.; L. C. Forti; C. R. F. Brandão; J. H. C. Delabie \& H. L. Vasconcelos. 1991. Ecologia nutricional de formigas. p: 131-223. In: A. R. Panizzi, \& J. R. P. Parra. Ecologia nutricional de insetos e suas implicações no manejo de pragas. São Paulo, Editora Manole Ltda. 359 p.

Harada, A. Y. \& J. Adis. 1997. The ant fauna of tree canopies in Central Amazonia: a first assessment, p. 382-400. In: N. E. Stork; J. Adis \& R. K. Didham (eds.). Canopy Arthropods. London, Chapman \& Hall, 567 p.

Harada, A. Y. \& J. Adis. 1998. Ants obtained from trees of a "Jacareúba" (Calophyllum brasiliense) forest plantation in Central Amazonian by canopy fogging: first results. Acta Amazônica 28: 309-318.

Heckman, C. W. 1998. The Pantanal of Poconé. Dordrecht, Kluwer Academic Plubishers, $622 \mathrm{p}$.

Hölldobler, B. \& E. O. Wilson. 1990. The ants. Berlin, SpringerVerlag. xii $+732 \mathrm{p}$.

Hurtado-Guerrero, J. C.; C. R. V. da Fonseca; P. M. Hammond \& N. E. Stork. 2003. Seasonal variation of canopy arthropods in Central Amazon, p. 170-175. In: Y. Basset; V. Novotny; S. E. Miller \& R. L. Kitching (eds.). Arthropods of tropical forests. Spatiotemporal dynamics and resource use in the canopy. Cambridge, Cambridge University Press, 574 p.

Kitching, R. L.; H. Mitchell; G. Morse \& G. Thebaud. 1997. Determinants of species richness in assemblages of canopy arthropods in rainforests, p. 131-150. In: N. E. Stork; J. Adis \& R. K. Didham (eds.). Canopy Arthropods. London, Chapman \& Hall, 567 p.

Lawton, J. H. 1983. Plant architecture and diversity of phytophagous insects. Annual Review Entomology 28: 23-29.

Longino J. T. \& N. M. Nadkarni. 1990. A comparison of ground and canopy leaf litter ants (Hymenoptera: Formicidae) in a Neotropical montane forest. Psyche 97: 81-94.

Lucky, A.; T. L. Erwin \& J. D. Witman. 2002. Temporal and espatial diversity and distribuition of arboreal Carabidae (Coleoptera) in a western Amazonian Rain forest. Biotropica 34: 376-386.

Magurran, A. E. 1988. Diversidad Ecológica y su Medición. Barcelona, Ediciones Vedra, 197 p.
Majer, J. D. \& J. H. C. Delabie. 1994. Comparison of the ant communities of annually inundated and terra firme forests at Trombetas in the brazilian Amazom. Insects Sociaux 41: 343359.

Marques, M. I.; J. Adis; C. Nunes Da Cunha \& G. B. Santos. 2001. Arthropod biodiversity in the canopy of Vochysia divergens Pohl (Vochysiaceae), a forest dominant in the Brazilian Pantanal. Studies on Neotropical Fauna and Environment 36: 205210.

Nadkarni, N. M. 1994. Diversity of species and interactions in the upper tree canopy of forest ecosystems. American Zoology 34: $70-78$.

Nadkarni N. M. \& J. T. Longino. 1990. Invertebrates in canopy and ground matter in a Neotropical montane Forest, Costa Rica. Biotropica 22: 286-289.

Samways, M. J. 1994. Insect Conservation Biology. London, Chapman \& Hall, 357p.

Santos, G. B.; M. I. Marques; J. Adis \& C. R. de Musis. 2003. Artrópodos associados à copa de Attalea phalerata Mart. (Arecaceae), na região do Pantanal de Poconé-MT. Revista Brasileira de Entomologia 47: 211-224

Stork, N. E. 1991. The composition of arthropod fauna of Bornean lowland rainforest trees. Journal of Tropical Ecology 7: 161180.

Tillberg, C. V. \& M. D. Breed. 2004. Placing an omnivore in a complex food web: dietary contributions to adult biomass of an ant. Biotropica 36: 266-271.

Tobin, J. E. 1995. Ecology and diversity of tropical forest canopy ants, p. 129-147. In: M. D. Lowmam, \& N. M. Nadkarni. 1995. Forest Canopies. San Diego, Academic Press, 624 p.

Vasconcelos, H. L. de 1990. Effects of litter collection by understorey palms on the associated macroinvertebrate fauna in Central Amazonia. Pedobiologia 34: 157-160.

Way, M. J. \& B. Bolton. 1997. Competition between ants for coconut palm nesting sites. Journal of Natural History 31: 439-455.

Watanabe, H. 1997. Estimation of arboreal and terrestrial arthropod densities in the forest canopy as measured by insecticide smoking, p. 401-413. In: N. E. Stork; J. Adis \& R. K. Didham (eds.). Canopy Arthropods. London, Chapman \& Hall, 567 p.

Wilson, E. O. 1986. The organization of flood evacuation in the ant genus Pheidole (Hymenoptera: Formicidae). Insects Sociaux 33: 458-469.

Wilson, E. O. 1987. The arboreal ant fauna of Peruvian Amazonian forests: a first assessment. Biotropica 19: 245-251. 